\title{
Power quality events detection using fourth-order spectra
}

\author{
Juan José González de la Rosa ${ }^{1}$, José María Sierra-Fernández ${ }^{1}$, \\ Agustín Agüera-Pérez ${ }^{1}$, José Carlos Palomares-Salas ${ }^{1}$, Antonio Moreno-Muñoz ${ }^{2}$ \\ ${ }^{1}$ Univ. of Cádiz. Electronics, Research Group PAIDI-TIC-168 \\ EPSA-Av. Ramón Puyol S/N, E-11202-Algeciras-Spain \\ Email: juanjose.delarosa@uca.es \\ ${ }^{2}$ Univ. of Córdoba-Spain. Electronics, Research Group PAIDI-TIC-168 \\ Campus Rabanales. Leonardo Da Vinci building \\ Email: amoreno@uco.es
}

\begin{abstract}
This paper introduces the use of a fourth-order frequency-domain statistical estimator, the spectral kurtosis (SK), in the field of power-quality analysis. The research has been organized in the frame of a research national project and points towards the implementation of these techniques into an automatic platform to perform $P Q$ analysis in power plants and power inverters. Higher-order statistics in the frequency domain manage to distinguish 3 types of electrical anomalies (sags, swells and transients), with an accuracy of $83 \%$.
\end{abstract}

Keywords-Higher-Order Statistics (HOS), Frequency domain analysis, Power Quality (PQ), Spectral Kurtosis (SK).

\section{INTRODUCTION}

The Spectral Kurtosis (SK) is roughly described as the kurtosis of each frequency component, and compares the variability in amplitude of the different Fourier frequencies. Then, this statistics indicates how the impulsiveness of a signal varies with frequency. Since Power-Quality (PQ) events give rise to sudden changes in the power line, this higher-order statistics is potentially useful to characterize the frequency bands associated to each type of electrical anomaly. Following this line, the goal of the paper is to use an estimator of the SK to measure the variability of each frequency component of the electrical signal. Consequently, a constant-amplitude (zero variability) single-frequency sinusoid exhibits a minimum SK at this frequency; where as if the amplitude varies with time the SK increases at this frequency. More precisely, if the amplitude varies according to a normal distribution, the SK is zero.

This philosophy has been formerly brought to practice in the time-domain by several notable works. Bollen et al. used advanced signal processing techniques to introduce new statistical features to PQ event detection [1]. Ribeiro et al. [2] used HOS to extract new time-domain features associated to electrical anomalies. Categorization of PQ events has been previously performed in the work [3] HOS techniques has also been implemented to specifically detect sags and swells [4].

The higher-order statistics have been used extensively during the past two decades. They were introduced in the eighties for detecting transients in sonar processing to enhance non-linearities, discovering hidden processes, due to their capability to reject noise and to complement the classical second-order characterization [5]. One of the most useful tools is the fourth-order cumulant, in which the kurtosis is inspired. Its complementary version in the frequency domain, is the so-called three-spectrum, and the SK constitutes a particular case, successfully used to distinguish different types of signals from their statistical properties [6]. Applications have been biased in machines' surveillance [7]-[9]; other works are found in the field of insect detection [10].

With respect to the worldwide interest in $\mathrm{PQ}$, a twofold objective is pursued. Equipment has become more sensitive to anomalies in the power-line, and at the same time the industrial electronic modules cause voltage disturbances. There is also the need for standardization and performance criteria for consumers and utilities. Consequently, and motivated by this issue a simple technique has been adopted in former works, with the goal of performing an on-site procedure, which was independent both from the device under test, as it uses normalization [4], [11]. PQ events are roughly of the same frequency of the 'healthy' signal (ideal power-line sine wave). Thus, signal processing techniques benefit from this fact by measuring-detecting changes in the statistical values as the target signal is tested. The traditional analysis procedure is based in the use of a sliding window through which the statistical estimator is computed. Thereby, when the perturbed zone in the signal appears (the sliding window bumps into the anomaly), a change in the estimator is observed and automatically targeted. This working hypothesis is also present in the present research. According to this perspective, this work presents performance results of SK over a battery of PQ events. Results are promising since the test offers an accuracy of $84 \%$ over an ensemble of real-life signals.

The paper is structured as follows. The following Section II, introduces the estimator for the SK and presents its performance over illustrative signals, with the goal of a better understanding. Results are presented in Section III, where a collection of synthetics and real-life electrical 
faults is presented. Finally, conclusions are drawn in Section IV.

\section{THE SPECTRAL KURTOSIS: DESCRIPTION AND ILLUSTRATIVE EXAMPLES}

This section aims to introduce SK and expose the theoretical foundation of the present research through examples. In statistics, kurtosis is a measure of the "peakedness" of the probability distribution of a realvalued random variable. Higher kurtosis means more of the variance is due to infrequent deviations, as opposed to frequent modestly-sized deviations. Kurtosis is more commonly defined as the fourth central cumulant divided by the square of the variance of the probability distribution, which is the so-called excess kurtosis, as elicited in Eq. (1):

$$
\gamma_{2}=\frac{\kappa_{4}}{\kappa_{2}^{2}}=\frac{\mu_{4}}{\sigma^{4}}-3
$$

where $\mu_{4}=\kappa_{4}+3 \kappa_{2}^{2}$ is the 4 th-order central moment; and $\kappa_{4}$ is the 4 th-order central cumulant, i.e. the ideal value of $\operatorname{Cum}_{4, x}(0,0,0)$. The "minus 3 " at the end of this formula is a correction to make zero the kurtosis of the normal distribution. Excess kurtosis ranges from -2 to $+\infty$. This definition of the 4 th-order cumulant for zero time-lags comes from a combinational relationship among the cumulants of stochastic signals and their moments, and is given by the Leonov-Shiryaev formula.

The sample kurtosis is calculated over a sample-register (an $N$-point data record), and noted by the Eq. (2):

$g_{2}=\frac{m_{4}}{s^{4}}-3=\frac{m_{4}}{m_{2}^{2}}-3=\frac{\frac{1}{N} \sum_{i=1}^{N}\left(x_{i}-\bar{x}\right)^{4}}{\frac{1}{N^{2}}\left[\sum_{i=1}^{N}\left(x_{i}-\bar{x}\right)^{2}\right]^{2}}-3$,

where $m_{4}$ is the fourth sample moment about the mean, $m_{2}$ is the second sample moment about the mean (that is, the sample variance), and $\bar{x}$ is the sample mean.

Ideally, the spectral kurtosis represents of the kurtosis of each frequency component of a process (or data from a measurement instrument $x_{i}$ ). For estimation issues we consider $M$ realizations of the process; each containing $N$ points; i.e. we consequently consider $M$ measurement sweeps, each sweep with $N$ points. The time spacing between points is the sampling period, $T_{s}$, of the data acquisition unit. The SK unbiased estimator is given by the Eq. (3):

$$
\widehat{G}_{2, X}^{N, M}=\frac{M}{M-1}\left[\frac{(M+1) \sum_{i=1}^{M}\left|X_{N}^{i}(m)\right|^{4}}{\left(\sum_{i=1}^{M}\left|X_{N}^{i}(m)\right|^{2}\right)^{2}}-2\right],
$$

where $m$ indicates the frequency index, and $\hat{G}_{2, X}^{N, M}$ indicates the value of the kurtosis for this Fourier frequency.

Whilst the present works focuses in the frequency domain. Promising time domain results have been obtained in [4]; research in which frame the signal analysis was performed using the initial hypothesis that the sliding

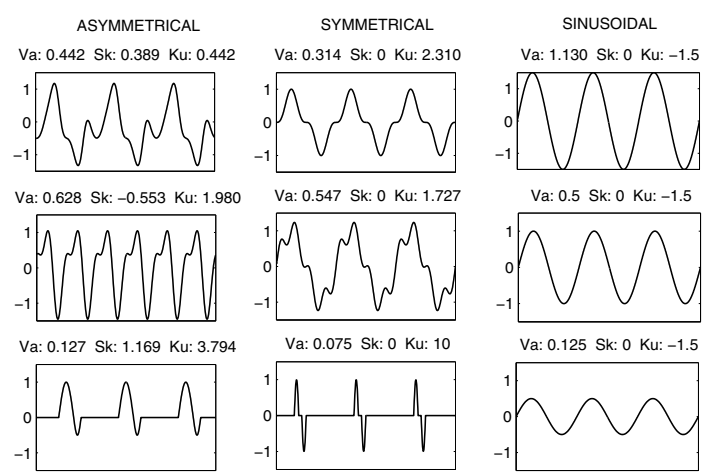

Fig. 1. Three common sets of anomalies revealing different constant values for the statistics.

window used to extract HOS features (statistics) enclosed an exact number of cycles of the $50 \mathrm{~Hz}$ sine wave. The analysis is based on windows of $0.02 \mathrm{~s}$ width, which covers one cycle of a standard power signal (healthy signal). Thus, by displacing the sliding window along a healthy signal, the set of values analyzed by the HOS processing is identical, returning a specific constant value for the statistical estimator. Any electrical disturbance or anomaly over the healthy signal would produce variations from this constant value when the sliding window bumped into this distortion in the waveform, thereby revealing its presence. Furthermore, if the coupled disturbance generated another $50 \mathrm{~Hz}$ signal, it returned constant values, which were different from the ones associated to the healthy power-line. Consequently, we benefit from this fact, to characterize the $50 \mathrm{~Hz}$ disturbances coupled to the power-line.

The benefit of the suggested procedure of analysis is that $50 \mathrm{~Hz}$ events are tuned by the sliding window, and the returned constant values are associated to the specific shapes of the waveforms. In order to show this working premise, an introductory example has been conceived. Nine $50-\mathrm{Hz}$ signals, grouped in three sets, have been analyzed in order to observe their associated constant values. These signals and their constant HOS values are represented in Fig. 1 classified in asymmetrical, symmetrical and pure-sinusoidal signals.

These results in the time-domain are complementary to achieve satisfactory interpretation in the frequency domain. An example in the frequency domain has been designed as a previous step. It benefits from noise rejection and at the same time, it illustrates the performance of the SK estimator over illustrative spectra. A number of 500 realizations (2,205 data)of two mixtures using a sample frequency of $44,100 \mathrm{~Hz}$, and its processing results presented in the Fig. 2. The left couple of graphs shows how random-Gaussian-amplitude sine wave $(5 \mathrm{kHz})$ is outlined in the SK graph by "1", considering the superimposed behaviors of the sinusoidal waveform and the random process; while the constant-amplitude sines (2, $9 \mathrm{kHz}$ ) are characterized by a "-1" in the SK graph with independence of their amplitudes' values. The second pair of graphs shows noise rejection for a colored Gaussian 
noise; besides it is seen that SK reaches a value between "0" and "-1", for the sine wave at $2 \mathrm{kHz}$.
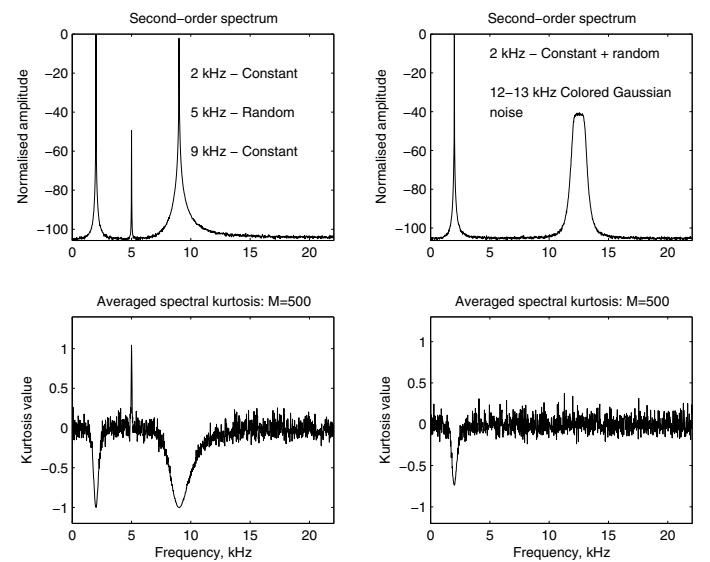

Fig. 2. Performance of the SK estimator over synthetics.

\section{CAse studies And Results}

Prior to the real-life situations, six cases have been analyzed corresponding to illustrative situations of electrical faults' detections. To optimize space, only one case is illustrated in this paper in a figure, which in turn contains five boxes or graphs. The upper box comprises the timedomain register, or raw data. Just below, the computation results of the variance and skewness are shown: second and third-order statistics, respectively. Finally, the timedomain kurtosis and the spectral kurtosis are displayed. Each case comprises a 20k-point synthetic time-domain register with a duration of $1 \mathrm{~s}(20 \mathrm{kHz}$ sampling frequency). An additive normal noise process (1\% of the amplitude of the signal) has been added in order to achieve a more realistic behavior.

The enactment of the research is described hereinafter. The time-domain statistics have been computed using a 400-point sliding window (which corresponds to a signal period). The sliding time (time shifting) has been fixed to 0.025 cycles, which corresponds to $0.5 \mathrm{~ms}$. First, the analysis of the sliding statistics in the time-domain is performed. It is observed that, during the normal operation and during the electrical anomaly the all three temporal estimators output a constant value (calculated over sliding windows); alterations are only observed during the transition between different zones of the time-domain signal. This is due to the fact that the temporal analysis has been performed with a $50-\mathrm{Hz}$-synchronized window. Although the $50-\mathrm{Hz}$ frequency remains constant, the amplitude changes, and subsequently the constant value of the statistic estimator also changes. The fact has been demonstrated in former works [4], [12] and constitutes, as previously explained, the working hypothesis.

To calculate the SK from the time-domain acquired data we extract realizations or segments with a length of 0.1 s, with an overlap of $50 \%$. This means a number of 19 segments for each raw data. Then the SK is averaged over $19,(1 / 0,05)-1$, segments plus 981 segments, which in turn correspond to healthy normalized power-line signals, that are stored in memory. Following this procedure we assure that the SK is averaged over a big enough number of segments. The 981 segments come from healthy parts which have been added before the defect. The defect is the only part of the signal which is displayed in the following graphs.

Fig. 3 shows voltage sag to $60 \%$ (residual voltage); i.e. the remaining amplitude of the signal is of $60 \%$, during the electrical anomaly; which means that the line voltage is reduced down to $60 \%$ of the normal value, It is observed a drastic reduction of the sliding variance in the anomaly zone in comparison to the normal condition value $(0.5)$. This is due to the fact that the variance is a measure of the instantaneous signal power. The higher the amplitude level, the greater the power.

The behavior of the sliding skewness (in the rightcentered graph) is quite different from the time-domain variance. While, during the anomaly, the signal exhibits the same skewness as in the normal situation (zero valued), in the transition zones two peaks or (bumps) appear. This is explained by the fact that the skewness is related to the signal symmetry, which it is preserved for this type of defect. But during the transition, the symmetry is lost in a fragment of cycle. During the first transition, the negative semi-cycle presents a higher amplitude value than the positive semi-cycle (time instant of $0.4 \mathrm{~s}$ in Fig. 3 ) then the skewness rapidly evolves towards negative values, showing a negative peak. In the second transition it happens the other way round; i.e., the positive semi-cycle offers a higher amplitude, so that the sliding skewness turns positive.

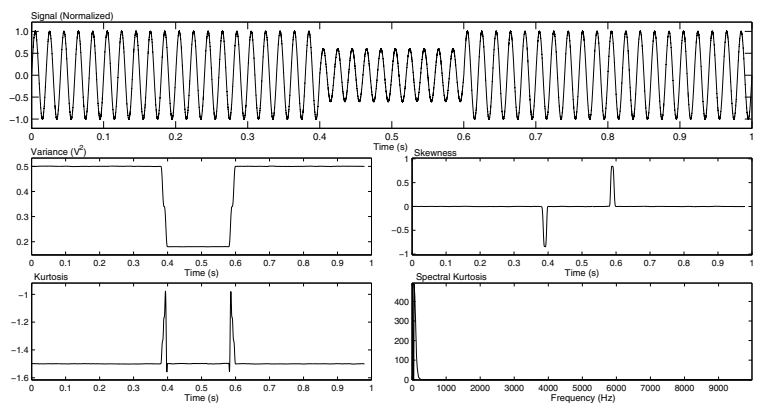

Fig. 3. A $40 \%$ voltage sag with a duration of $0.2 \mathrm{~s}$.

The kurtosis behavior's in the time domain (in the lower-left graph of Fig. 3), confirms the results derived from the skewness' analysis. The kurtosis is associated to the waveform's shape of the register, which does not vary during the electrical anomaly (during the defect the symmetry also remained constant). Two peaks are observed with the same polarity. This is due to the fact that, although the evolution of the amplitude values are different for both transitions (high-to-low vs. lowto-high) the kurtosis identifies the deformation itself. In the moment, the deformation has turned to a constant amplitude value, the sliding kurtosis returns to its normal vale $(-1,5$ steady value $)$. 
In the frequency domain, the SK (in the lower-right graph of Fig. 3) offers a zero value for all the range, with the exception of the frequencies below $500 \mathrm{~Hz}$, characterized by a general increase, which denotes the changes in the amplitude. Intriguingly, making a zoom of this region of the spectrum allows the observation of a -1 valued peak for the $50-\mathrm{Hz}$ component, associated to the constant amplitude.

It is true that the amplitude varies; and in fact this is the definition of the electrical anomaly, but the SK considers a long enough period of the waveform, so that an alteration of 0.2 seconds vs. the rest of the duration $(100 \mathrm{~s})$ of the healthy signal does not affect the result. The rest of the spectrum presents a zero value (or a value towards zero), associated to the frequencies that exhibit a Gaussian behavior.

The real test to corroborate the results from the synthetics' analysis, has been developed using real-life signals, available from the IEEE working group P1159.3 web site. These signals have been originally sampled at $15,360 \mathrm{~Hz}$ and correspond to $60 \mathrm{~Hz}$ events. In order to adapt this power-line frequency to the $50 \mathrm{~Hz}$ European network, we have re-calculated the sampling frequency to $12,800 \mathrm{~Hz}$ $(15,360 \cdot 50 / 60)$.

Then each 0.12 s-length register, contains $12,800 \cdot 0.12=1,536$ data. In comparison to the former set of synthetics, now the horizontal axis of the SK expands to $6,400 \mathrm{~Hz}$ (sampling frequency $12,800 \mathrm{~Hz}$ ). Windows are overlapped $50 \%$, and three different lengths have selected for the SK realizations to be averaged (0.02, 0.04 and $0.06 \mathrm{~s}$ ). The final $\mathrm{SK}$ graph is the result of averaging 1,000 realizations or segments using the estimator. For example, considering the less resolution length, $(0.02 \mathrm{~s})$ there are (0.12/0.01)-1 segments extracted from the time domain data. As the averaging is performed over 1,000 realizations, 989 more healthy realizations are added.

On the other hand, as the sampling rate is $12,800 \mathrm{~Hz}$, a 0.02-s realization contains 256 samples. Consequently, there are 128 data in the SK graph, with a frequency resolution of $50 \mathrm{~Hz}(6,400 \mathrm{~Hz} / 128$ data). Despite this fact, the SK provides the user with the same information as in the case of the synthetics, this time gaining computation speed. The SK result is also averaged over 1,000 segments, as in the case of the synthetics.

The first real-life case consists of a sag which starts with a transient. The presence of this transient is denoted by the rebounds at the starting point of the defect, as shown in Fig. 4. In the time-domain (the upper subgraph in Fig. 4) the variance presents a value of 0.5 in the first cycle, and then it goes to almost zero as the total power vanishes. The skewness' and kurtosis' behaviors are explained similarly as in the cases of the synthetics, indicating the symmetry and the waveform shape, respectively. During the defect, the time-domain signal is almost sinusoidal, giving rise to a zero kurtosis.

Two significative maxima, at the frequencies $950 \mathrm{~Hz}$ and $2,100 \mathrm{~Hz}$, are observed in the SK graph, corresponding to this transient. The initial region in the spectrum matches with the results obtained in the theoretical case for the sag situation. The SK graph corresponds to a resolution of $0,02 \mathrm{~s}$.

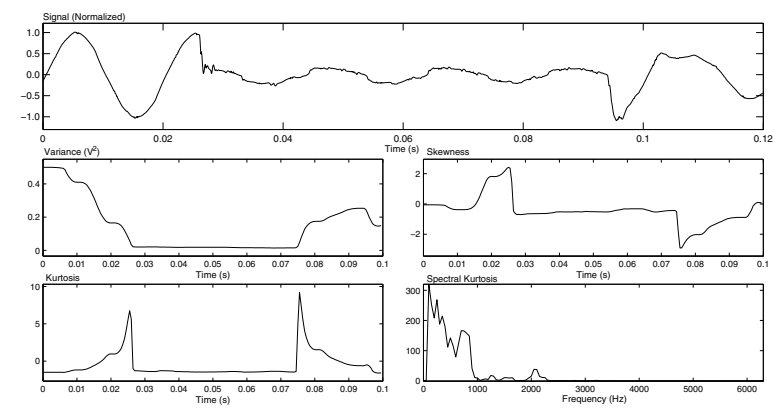

Fig. 4. Real case \#1: A sag plus an oscillatory transient, caused by a short circuit. Two peaks appear at 950 and $2,100 \mathrm{~Hz}$ indicating the transient's features in the frequency domain. SK: time resolution=0,02 $\mathrm{s}$; frequency resolution $=50 \mathrm{~Hz}$.

Different resolutions in the SK may lead to very different SK patterns. In this case, higher-resolution spectra do not offer the same results, as it is proved in Fig. 5 , because if the time-window is wider, the power of the transient returned by the FFT will be less, as it is averaged. Intriguingly, the peaks in 950 and $2,100 \mathrm{~Hz}$ vanish for wider window's.

The second practical case is depicted in Fig. 6 and consists of a sag ending with harmonics. This anomaly comes from the recovery process that is experimented by a network after a disconnection of a malfunctioning load. The variance indicates a power recovery from the minimum value. At the instant 0.02 s., the sliding skewness begins to indicate the symmetry's deviation. The kurtosis suddenly goes up, indicating the drastic change in shape. The behavior of the skewness and the kurtosis is the same as in the theoretical cases, for this type of defects.

The SK graph only reveals the presence of the sag, indicated by the bump in the low frequency range. During the sag, there is an oscillation at $500 \mathrm{~Hz}$. It is not possible to discriminate the harmonics, because of the low resolution, $50 \mathrm{~Hz}(0.02 \mathrm{~s})$.

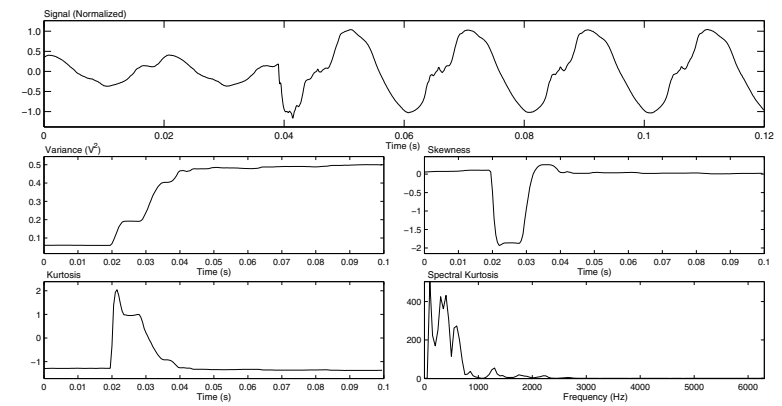

Fig. 6. Real case \#2: A sag plus an oscillatory transient, caused by a disconnection of a malfunctioning load (short circuit or earth fault). $\mathrm{SK}$ : time resolution $=0,02 \mathrm{~s}$; frequency resolution $=50 \mathrm{~Hz}$.

If we compare the three resolution cases, as in Fig. 7, the harmonics can be seen at the components 100,150 , 200 and $250 \mathrm{~Hz}$, in the graphs corresponding to 0.04 and $0.06 \mathrm{~s}$. 
- The third real case is presented in Fig. 8, and consists of a sag containing an oscillatory transient, which may be caused by a capacitors battery. The variance's behavior reduces its nominal value, as it is expected from the power reduction. During the defect, the symmetry is lost, due to the presence of transients. At the end of the defect, the signal has less energy (as indicated from the sliding variance) but the symmetry is recovered. The timedomain kurtosis begins in -1,5 (the nominal value) and then it evolves to higher values that indicates that the sinusoidal waveform is lost. During the defect, the value is slightly higher than $-1,5$, indicating the presence of a quasi-sinusoidal waveform.

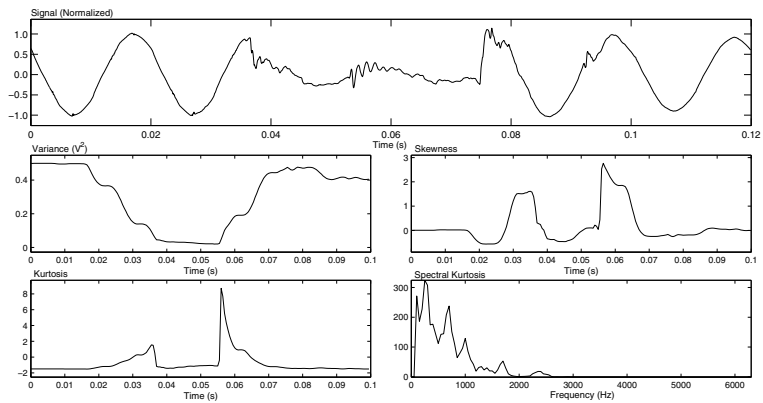

Fig. 8. Real case \#3: A sag plus an oscillatory transient $(750 \mathrm{~Hz})$, caused by a capacitors battery. SK: time resolution $=0,02 \mathrm{~s}$; frequency resolution $=50 \mathrm{~Hz}$.

The oscillatory transient at $750 \mathrm{~Hz}$ is present in the three SK graphs of Fig. 9; in fact resolution improves the detection of this frequency component.

The procedure has been tested using 50 real-life signals, obtaining an $84 \%$ of accuracy. The false positives are due to the per se oscillatory behavior of the power line signal.

\section{CONCLUSIONS}

This paper was devoted to use the spectral kurtosis (a fourth-order spectrum) with the goal of detecting PQ events, as an alternative to time-domain methods and second order spectra (power spectrum). The theoretical definition and comprehensive examples have been introduced in order to get full understanding of the PQ event targeting process via the SK. Real-life situations demonstrate its effective practical use, since results are correlated to theoretical synthetics.

The SK estimator is noise tolerant, behaving as expected up to a $\mathrm{SNR}=35 \mathrm{~dB}$. A practical approach has been performed using real-life signals from the IEEE working group P1159.3. Under real conditions the algorithm behaves properly with an accuracy of the $84 \%$. The results obtained for this application are encouraging, but some improvements (more tests) are still to be performed in order to transfer the method to an automatic detection system. This off-line stage can be considered as finished.

Grace to the estimator's normalization, this fourth-order statistics is independent of the signal power, providing additional information to the traditional obtained from the power spectral density. As a future work it would be desirable to test the procedure in several electrical power plants, and inverters in order to get a full testing branch. This would only suppose to get data, as the estimator is power-independent, and the research conveys the need for a close cooperation between power-system researchers and network or grid operators.

Finally, it is necessary to mention that false positive cases are mainly due to the effects of frequency shifts from the nominal value.

\section{ACKNOWLEDGEMENT}

The authors would like to thank the Spanish Government for funding the research project TEC2010-19242C03-03 (SIDER-HOSAPQ). Our unforgettable thanks to the trust we have from the Andalusian Government for funding the Research Group PAIDI-TIC-168 in Computational Instrumentation and Industrial Electronics (ICEI).

\section{REFERENCES}

[1] M. H. J. Bollen, I. Y.-H. Gu, P. G. V. Axelberg, and E. Styvaktakis, "Classification of underlying causes of power quality disturbances: Deterministic versus statistical methods," EURASIP Journal on Advances in Signal Processing, vol. 2007, no. Article ID-79747, pp. 1-17, 2007.

[2] M. V. Ribeiro, C. A. G. Marques, C. A. Duque, A. S. Cerqueira, and J. L. R. Pereira, "Detection of disturbances in voltage signals for power quality analysis using hos," EURASIP Journal on Advances in Signal Processing, vol. 2007, no. Article ID-59786, pp. 1-13, 2007.

[3] Omer Nezih Gerek and D. G. Ece, "Power-quality event analysis using higher order cumulants and quadratic classifiers," IEEE Transactions on Power Delivery, vol. 21, no. 2, pp. 883-889, April 2006

[4] A. Agüera-Pérez, J. C. P. Salas, J. J. G. De la Rosa, J. M. SierraFernández, D. Ayora-Sedeño, and A. Moreno-Muñoz, "Characterization of electrical sags and swells using higher-order statistical estimators," Measurement (Ed. Elsevier), vol. 44, no. Issue 8, pp. 1453-1460, October 2011.

[5] A. K. Nandi, Blind Estimation using Higher-Order Statistics, 1st ed., A. K. Nandi, Ed. Boston: Kluwer Academic Publishers, 1999, vol. 1, no. 1.

[6] V. Vrabie, P. Granjon, and C. Serviere, "Spectral kurtosis: from definition to application," in IEEE-EURASIP International Workshop on Nonlinear Signal and Image Processing (NSIP'2003), IEEE, Ed., vol. 1, no. 1, Grado, Italy on June 8-11, 2003 2003, pp. 1-5.

[7] D. Ho and R. Randall, "Optimisation of bearing diagnostic techniques using simulated and actual bering fault signals," Mechanical Systems and Signal Processing, vol. 14, no. 5, pp. 763-788, september 2000.

[8] N. Sawalhi and R. B. Randall, "The applicacion of spectral kurtosis to bearing diagnostics," in Proceedings of the 2004 AAS meeting, A. A. Society, Ed., vol. 1, no. 1, Gold Coast, Australia, November 3-5 2004, pp. 1-5.

[9] J. Antoni, "The spectral kurtosis: application to the vibratory surveillance and diagnostics of rotating machines," Mechanical Systems and Signal Processing (Ed. Elsevier), vol. 20, no. 2, pp. 308-331, february 2006.

[10] J. J. G. De la Rosa and A. M. Muñoz, "Higher-order cumulants and spectral kurtosis for early detection of subterranean termites," Mechanical Systems and Signal Processing (Ed. Elsevier), vol. 22, no. Issue 1, pp. 279-294, February 2008, available online 1 September 2007.

[11] J. J. G. De la Rosa, A. Agüera-Pérez, J. C. P. Salas, J. M. SierraFernández, and A. Moreno-Muñoz, "A novel virtual instrument for power quality surveillance based in higher-order statistics and case-based reasoning," Measurement (Ed. Elsevier), vol. 45, no. Issue 7, p. 18241835, August 2012.

[12] J. J. G. De la Rosa, J. C. P. Salas, A. Agüera-Pérez, J. M. SierraFernández, and A. Moreno-Muñoz, "A novel virtual instrument for power quality surveillance based in higher-order statistics and case-based reasoning," Measurement (Ed. Elsevier), vol. 44, no. Issue 8, pp. 1453-1460, October 2011. 

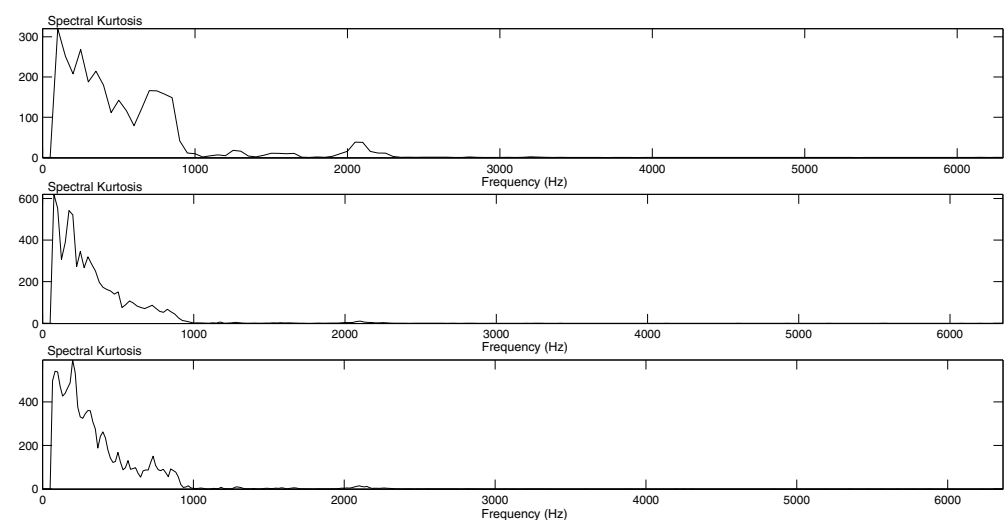

Fig. 5. Real case \#1: Comparison of the SK's graphs averaged for the time resolutions 0,02, 0,04 and 0,06 s, respectively from up to down.
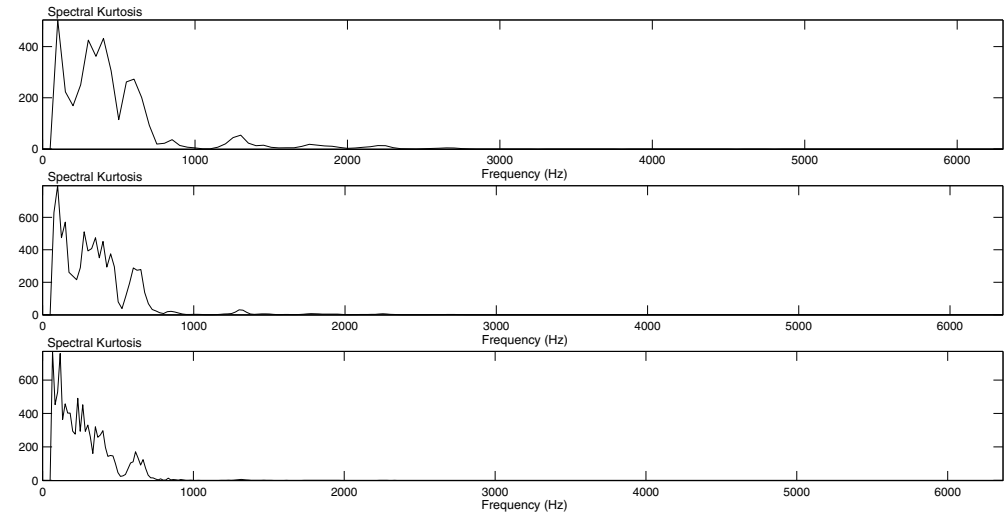

Fig. 7. Real case \#2: Comparison of the SK's graphs for the resolutions 0.02, 0.04 and 0.06 s., respectively up to down.
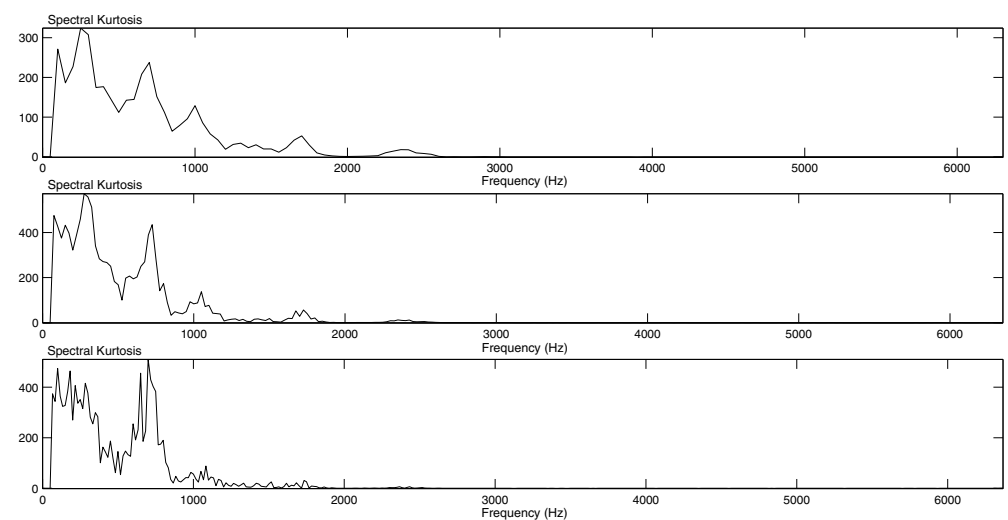

Fig. 9. Real case \#3: Comparison of the SK's graphs for the time resolutions $0.02,0.04$ and 0.06 s., respectively from up to down. The transient at $750 \mathrm{~Hz}$ is clearly outlined for the three resolution cases. 